\title{
Design and optimization of a monolithically integratable InP-based optical waveguide isolator
}

\author{
Mathias Vanwolleghem, Philippe Gogol, and Pierre Beauvillain \\ Institut d'Electronique Fondamentale, Université Paris-Sud, Bâtiment 220, 91405 Orsay Cedex, France \\ Wouter Van Parys and Roel Baets \\ Department of Information Technology, Faculty of Engineering, Ghent University, St-Pietersnieuwstraat 41 , \\ 9000 Gent, Belgium
}

Received June 23, 2006; accepted August 2, 2006;

posted September 11, 2006 (Doc. ID 72317); published December 20, 2006

\begin{abstract}
The optimization design of the layer structure for a novel type of a $1.3 \mu \mathrm{m}$ monolithically integrated InP-based optical waveguide isolator is presented. The concept of this component is based on introducing a nonreciprocal loss-gain behavior in a standard semiconductor optical amplifier (SOA) structure by contacting the SOA with a transversely magnetized ferromagnetic metal contact, sufficiently close to the guiding and amplifying core of the SOA. The thus induced nonreciprocal complex transverse Kerr shift on the effective index of the guided TM modes, combined with a proper current injection, allows for forward transparency and backward optical extinction. We introduce two different optimization criteria for finding the optimal SOA layer structure, using two different figure-of-merit functions (FoM) for the device performance. The device performance is also compared for three different compositions of the $\mathrm{Co}_{x} \mathrm{Fe}_{1-x}(x=0,50,90)$ ferromagnetic transition metal alloy system. It is found that equiatomic (or quasi-equiatomic) CoFe alloys are the most suitable for this application. Depending on the used FoM, two technologically practical designs are proposed for a truly monolithically integrated optical waveguide isolator. It is also shown that these designs are robust with respect to variations in layer thicknesses and wavelength. Finally, we have derived an analytical expression that gives a better insight in the limit performance of a ferromagnetic metal-clad SOA-isolator in terms of metal parameters. (C) 2006 Optical Society of America
\end{abstract}

OCIS codes: 250.5300, 230.3240, 230.3810, 160.3820.

\section{INTRODUCTION}

An optical isolator is an indispensable part of a modern optical telecom laser package. By eliminating accidental optical feedback into the laser diode, it suppresses one of the most important sources of intensity and wavelength noise. Commercial optical isolators are free-space bulk components, whose incorporation in the laser package necessitates precise alignment techniques that have an impact on the total cost of the laser package that greatly exceeds the cost of the isolator itself. A planar waveguidebased version of an optical isolator has therefore been a long-time pursued objective in the field of photonics. Next to that, a fully integrated laser-isolator component will obviously increase the mechanical and thermal stability of the laser package.

Traditional research toward integrated optical waveguide isolators has been focusing on using magneto-optic (MO) ferrimagnetic iron garnet materials in order to induce optical nonreciprocity in the component. The interest in this class of materials comes from their unique combination of low optical loss at telecom wavelengths and a considerably strong MO effect, the source of the nonreciprocity. Stand-alone devices with good isolation performance have been reported. ${ }^{1}$ The integration with a III-V host material, however, remains an issue. So far, the best reported isolation using garnet integrated on a III-V host did not exceed $5 \mathrm{~dB}$ in a device several millimeters long. ${ }^{2}$
An entirely different integration scheme has been theoretically proposed in 1999 almost simultaneously by Zaets and Ando ${ }^{3}$ and by Takenaka and Nakano. ${ }^{4}$ Their idea is to obtain straightforward monolithic integration of the isolator section by giving it an active III-V diodelike layer structure similar to the laser diode it needs to protect. They have shown that when such a semiconductor optical amplifier (SOA) structure is contacted by a transversely magnetized ferromagnetic metal contact sufficiently close to its guiding core layer, the complex transverse MO Kerr effect induces a nonreciprocal shift of the complex effective index of the guided TM modes. In other words, the modal absorption is different in both propagation directions. The remaining loss in the forward direction can be compensated for by current injection in the active material. The resulting component is essentially a nonreciprocal InP-based SOA pumped to transparency in one direction while remaining absorbing in the opposite direction, and being at the same time straightforwardly monolithically integrated with any InP-based active photonic device.

It has to be mentioned that the use of ferromagnetic metals on top of a standard semiconductor optical amplifier was originally theoretically proposed by Hammer et $a l .{ }^{5}$ However, these authors only considered nonreciprocal Faraday rotation in a ferromagnetic metal-clad amplifier. They also did not consider the important effects of ellip- 
ticity due to the strong MO metal dichroism. The MO metal-clad amplifier device as first proposed by Takenaka and by Zaets improves on this work, as it explicitly not only takes the metal dichroism into account but also bases its operation principle exclusively on this nonreciprocal absorption. Hence it is not hindered by the need for phase matching between TE and TM modes.

In 2004, Vanwolleghem et al. reported the first worldwide experimental evidence of this integrated isolator concept $^{6}$ using a $\mathrm{Co}_{90} \mathrm{Fe}_{10}$-contacted $1.3 \mu \mathrm{m}$ tensilestrained MQW InGaAsP/InP SOA, and recently Van Parys et al. presented devices with considerably improved performance. ${ }^{7}$ Using an equiatomic alloy composition $\mathrm{Co}_{50} \mathrm{Fe}_{50}$ and a tensile-strained MQW InAlGaAs/InP SOA structure, they have realized devices showing up to $99 \mathrm{~dB} / \mathrm{cm}$ isolation extinction at a reasonable transparency current density of $10 \mathrm{kA} / \mathrm{cm}^{2}$. In a previous paper, ${ }^{8}$ we reported, in detail, the derivation and the validity of the theoretical models that we developed to simulate this type of transverse MO nonreciprocal waveguides. It has been proven there that, apart from limit configurations that are merely of academic importance, a perturbation theory-based waveguide model, treating the nonreciprocal MO contribution to the TM waveguide dispersion equation as a first-order perturbation, achieves sufficient accuracy for the modeling of the behavior of this type of integrated optical waveguide isolator. An indication has been given of a possible optimization design strategy for this type of device.

In this paper, we present a more detailed and realistic treatment of the optimization of the SOA layer structure. In Section 2, the operation principle of the novel concept is concisely reviewed, supplemented, where available, with the characterized behavior of the different device building blocks. In Section 3, the different optimization methods are elaborated on, and some details of the simulation model are indicated. In Subsection 3.C, the simulation results are detailed. Section 4 presents a more indepth discussion of these results. A more physical explanation of the observed tendencies will be given, indicating what physical factors limit the ideal maximization of the nonreciprocal effect.

\section{NONRECIPROCAL FERROMAGNETIC- METAL-CONTACTED SEMICONDUCTOR OPTICAL AMPLIFIER: BASICS}

\section{A. Operation Principle}

At optical frequencies, a MO material is phenomenologically described by the vacuum magnetic permeability, $\mu_{0}$, and an antisymmetric (due to its nonreciprocity) tensorial electric permittivity $\epsilon$, whose nonzero off-diagonal elements are determined by the direction cosines of the magnetization $\mathbf{M}$ of the material. ${ }^{9}$

$$
\underline{\epsilon}_{\mathrm{MO}}=\epsilon_{0}\left[\begin{array}{ccc}
\epsilon_{r} & +j g \cos \left(\vartheta_{z}\right) & -j g \cos \left(\vartheta_{y}\right) \\
-j g \cos \left(\vartheta_{z}\right) & \epsilon_{r} & +j g \cos \left(\vartheta_{x}\right) \\
+j g \cos \left(\vartheta_{y}\right) & -j g \cos \left(\vartheta_{x}\right) & \epsilon_{r}
\end{array}\right] .
$$

Here, $\epsilon_{r}$ denotes the complex scalar isotropic dielectric constant, and $g$ denotes the complex gyrotropy (more specifically gyroelectric) constant, which is necessarily an odd first-order function of the magnetization $\mathbf{M}$, due to Onsager's principle $\left(\epsilon_{i j}(\mathbf{M})=\epsilon_{j i}(-\mathbf{M})\right)$. Due to the same principle, the contributions of the magnetization on the diagonal elements of the permittivity are necessarily of second order and are therefore in most cases negligible. When the MO material is lossless, the electric permittivity tensor has to be Hermitian, and both $\epsilon_{r}$ and $g$ will be purely real. For lossy MO materials, both $\epsilon_{r}$ and $g$ will be complex numbers. Using the $\exp (+j \omega t)$ sign convention, a lossy MO material can only take values for $\epsilon_{r}$ in the lower half of the complex plane, while for the gyroelectric parameter $g$, any value in the complex plane is physically feasible.

If the Cartesian coordinate system is chosen in such a way that the $z$ axis denotes the propagation direction of the waveguide and with the $x$ axis perpendicular to the epitaxial layer interfaces, Eq. (1) shows that when a transversely magnetized MO layer (i.e., along the $y$ axis, or thus $\vartheta_{x}, \vartheta_{z}=\pi / 2$ ) is incorporated in the waveguide, there will appear a nonreciprocal coupling (due to the antisymmetry of the off-diagonal $\epsilon_{\mathrm{MO}}$ elements) between the $E_{x}$ and $E_{z}$ components of the waveguide modes. Taking into account that for most photonic integrated circuits, the semivectorial approach of solving the Helmholtz equation is valid, ${ }^{10}$ this transverse MO waveguide effect will induce a propagation direction-dependent correction of the effective index of the guided TM modes (whose $E_{y}$ $\approx 0$ ). The TE spectrum (with $E_{x} \approx 0$ ) of the waveguide is unaffected. In a first approximation, this nonreciprocal correction (known as the transverse Kerr effect) of the TM effective index is proportional to the gyrotropy constant $g$ of the MO material (and an overlap integral of the TM modal profile with the MO layer). ${ }^{8}$ As a result, if the MO layer has loss and thus $g$ is complex, both the propagation constant and the modal loss of the guided TM modes will be different for forward and backward propagation through the waveguide. Exploiting this modal dichroic effect for the guided TM modes is the basic idea behind the proposed integrated optical waveguide isolator concept.

Its schematic layout and operation principle are sketched in Fig. 1. A transversely magnetized MO metal is incorporated sufficiently close to the guiding layer of a III-V semiconductor waveguide. The lossy character of the MO layer $(\Im(g) \neq 0)$ will cause the optical absorption of the guided TM modes to be different in opposite propagation directions of the waveguide. For such a device to behave truly as an optical isolator, it has to be supplemented with a way of compensating for the remaining loss in the forward direction caused by the metallic absorption. This can be done by giving the III-V semiconductor waveguide an active SOA layout and using the transversely magnetized MO metal contact also as an electric contact for the underlying structure. By proper current injection, the device can be biased to optical transparency in one direction, while the nonreciprocal transverse Kerr absorption will cause an effective optical absorption in the opposite direction.

The advantages of this isolator concept are obvious. The nonreciprocal effect acts directly on the modal ab- 
(a)
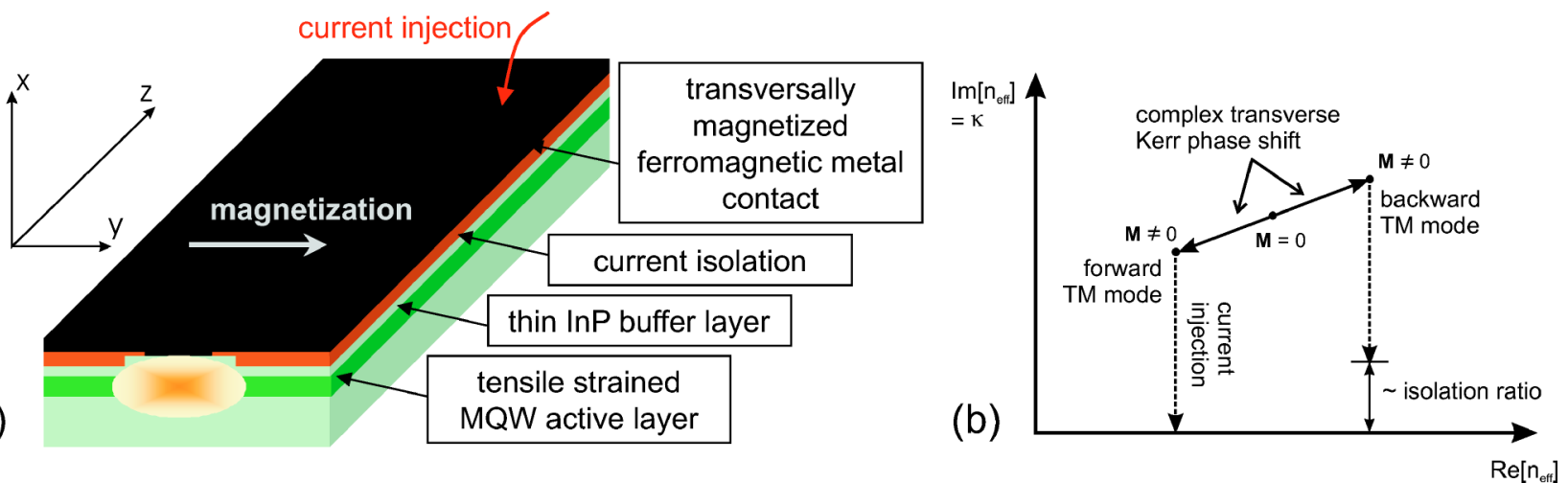

Fig. 1. (Color online) (a) Schematic and (b) operation principle of the monolithic integrated optical waveguide isolator.

Table 1. Measured Optical and MO Indices of the Three Studied CoFe Alloy Compositions at $1300 \mathbf{n m}^{a}$

\begin{tabular}{lccccc}
\hline Composition & $n$ & $\kappa$ & $g^{\prime}$ & $g^{\prime \prime}$ & $g / \epsilon$ \\
\hline $\mathrm{Co}_{90} \mathrm{Fe}_{10}$ & $4.65 \pm 0.1$ & $4.82 \pm 0.03$ & $-1.7 \pm 0.2$ & $-1.05 \pm 0.1$ & $-0.0220-0.0387 j$ \\
$\mathrm{Co}_{50} \mathrm{Fe}_{50}$ & $3.2 \pm 0.08$ & $4.5 \pm 0.04$ & $-1.7 \pm 0.2$ & $-1.7 \pm 0.1$ & $-0.0344-0.0710 j$ \\
$\mathrm{Fe}$ & $3.82 \pm 0.27$ & $3.5 \pm 0.05$ & $-1.4 \pm 0.3$ & $-0.75 \pm 0.17$ & $-0.0324-0.0495 j$ \\
\hline
\end{tabular}

${ }^{a}$ The last column gives the complex ratio of the metal dielectric constant and the metal gyrotropy, $g / \epsilon$. Zayets and Ando (Ref. 3 ) prove that the MO terms in the rigorous TM MO waveguide dispersion equation are proportional to this term.

sorption of the guided modes. Therefore there is no need for interferometric schemes as in garnet-based devices (see, for instance, Refs. 11 and 12). The ferromagnetic contact can be sputter deposited with controlled in-plane magnetic anisotropy so that the remanence in the transverse direction is sufficiently high and there is no need for an external magnet [as opposed to yttrium-iron-garnet(YIG) based devices]. Finally and most importantly, since the proposed device has basically a standard III-V SOA layer structure (except for the thinner cladding and the metal contact), this optical isolator can be straightforwardly monolithically integrated with a III-V semiconductor laser diode. Not only can it be grown on the same substrate, it can even have the same active layer structure.

\section{B. Building Blocks}

The experimental success of this device heavily depends on the strength of the SOA active region and on the electrical, magnetic and MO behavior of the ferromagnetic metal contact. We have reported the experimental results of our efforts to optimize the behavior of these building blocks for this specific application elsewhere. We suffice here by repeating those results that form the restricting boundary conditions for the optimization design of the device or that provide indispensable numerical input parameters for the calculation.

All of our work has been based on the use of the CoFe material system as the ferromagnetic contact material. In Ref. 13, we reported the possibility of obtaining an ohmic $p$-type $\mathrm{CoFe}$-based contact on $\operatorname{InGaAs}(\mathrm{P})$ with a sufficiently low contact resistivity. A special hybrid semiconductor contact layer structure needed to be designed in order to avoid too high levels of optical shielding of the MO metal. Details of this structure can be found in the mentioned reference and in Table 2 . It is important to underline that the presence of this bilayer is necessary for a good electrical behavior of the proposed device, and its properties can henceforth not be varied in the optimization design.

In Ref. 14, we reported optical and MO characterization of three $\mathrm{Co}_{x} \mathrm{Fe}_{1-x}$ alloy compositions $(x=0,50,90)$ using a generalized MO ellipsometer at a wavelength of $1300 \mathrm{~nm}$. The obtained values for the complex refractive indices and the complex gyrotropy constant are tabulated in Table 1 . These obviously serve as important input data for the optimization calculations presented here.

It is important to underline that the $\exp (+j \omega t-j \beta z)$ convention (with $\beta$ the propagation constant of the guided mode), and the preferred sign scheme of Atkinson and Lissberger is used in all optical and MO characterization experiments $^{15}$ and in all theoretical simulations. This implies the following relative signs for the real and imaginary parts of the indices,

$$
\begin{aligned}
& \epsilon_{r}=\epsilon_{r}^{\prime}-j \epsilon_{r}^{\prime \prime}=(n-j \kappa)^{2}, \\
& g=g^{\prime}-j g^{\prime \prime},
\end{aligned}
$$

where $n$ and $\kappa$ denote, respectively, the refractive index and the extinction coefficient. From the tabulated values, it seems obvious that the equiatomic alloy composition has the strongest MO properties. However, this does not automatically imply that it will be the best suited for the studied application. How the optimization of the device is governed by an interplay of all four indices will be shown in Section 4. To compensate for the remaining optical loss in the low-loss direction of the ferromagnetic metal-clad SOA, a high TM material gain tensile-strained multiple quantum well (MQW) active region has been designed and fabricated. Such a polarization discriminating gain 
region is necessary since the proposed isolator scheme only works for the TM polarization. In Ref. 16, our coworkers reported record TM gain values at $1300 \mathrm{~nm}$ by using tensile-strained InAlGaAs quantum wells (QWs). This optimized active core is composed of $10 \mathrm{~nm}$ thick, $-1.16 \%$ tensile-strained InGaAlAs QWs. They are separated by $20 \mathrm{~nm}$ thick, compressively strained (+0.64\%) InGaAlAs barriers $\left(\lambda_{g}=1.1 \mu \mathrm{m}\right)$. The MQW region is surrounded by lattice-matched InGaAsP $\left(\lambda_{g}=1.0 \mu \mathrm{m}\right)$ separate confinement heterostructure $(\mathrm{SCH})$ layers. The composition of these latter guiding layers has been optimized for an efficient carrier injection and is therefore also fixed in the optimization design of the SOA-isolator layer structure. Experimental determination of the modal gaincurrent density relation of the active material was done by threshold current density measurements on six QWs broad area as-cleaved Fabry-Perot lasers of varying length and thus varying mirror losses. In combination with the calculated $6 \mathrm{QW}$ TM confinement $\left(\Gamma_{\mathrm{TM}, 6 \mathrm{QW}}\right.$ $=0.14$ ) and the separately measured internal cavity loss, the modal gain at laser threshold can be evaluated. Figure 2 plots the measured TM modal gain as a function of current density.

A standard logarithmic gain-current relationship can be fitted to these measurement points leading to the following first-order approximation for the fundamental QW material gain $G_{\text {mat }}$ (in units of $1 / \mathrm{cm}$ ) as a function of total injected current density,

$$
G_{\mathrm{mat}}=\frac{66}{\Gamma_{\mathrm{TM}, 6 \mathrm{QW}}} \ln \left(\frac{6}{N_{w}} \frac{J_{\mathrm{tot}}}{0.35}\right)=471 \ln \left(\frac{6}{N_{w}} \frac{J_{\mathrm{tot}}}{0.35}\right),
$$

with $N_{w}$ as the number of wells in the MQW core and $J_{\text {tot }}$ as the total injected current density (expressed in $\mathrm{kA} / \mathrm{cm}^{2}$ ), Equation (3) obviously assumes that the injection and internal quantum efficiency are independent of the number of wells, and that the carrier concentration is uniformly distributed over the wells. This is a safe assumption for a number of wells not much exceeding ten. This experimentally determined gain-current relationship is used as a numerical boundary condition in the optimization design, namely, for the minimization of the needed injected current density for forward transparency.

\section{OPTIMIZATION DESIGN}

A. Device Structure-Nonreciprocal Waveguide Model With the above-described restrictions on the layer structure of the SOA-isolator, the remaining degrees of free-

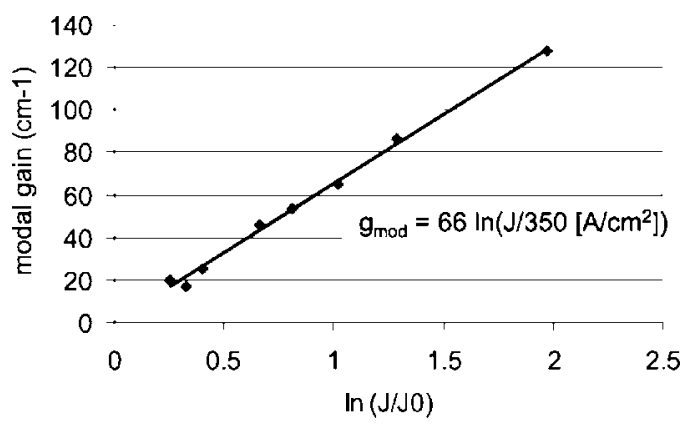

Fig. 2. Experimentally determined logarithmic gain-current relationship of the tensile-strained $6 \mathrm{QW}$ core (at room temperature.) dom for the optimization design are the thicknesses of both SCH InGaAsP layers and the thickness of the InP buffer layer between the amplifying core and the semiconductor contact layer. In other words, optimizing the layout of the SOA-isolator is mainly a question of finding the optimal transversal layer stack or thus optimizing a slab waveguide. It is the transversal modal behavior that will determine the optical confinement near the metal. The slab waveguide to be optimized is the one detailed in Table 2. The number of wells has not been varied in the optimization calculations. This is because it was not entirely experimentally clear whether at a higher number of wells one would not risk strain relaxation and structural defects as a result of the increasing amount of stored elastic energy. On the other hand, there have been indications that above ten wells, the internal quantum efficiency (for radiative recombination) of the MQW region might steeply drop due to an asymmetric carrier filling of the wells (because of the different mobilities of holes and electrons). Next to that, first calculations immediately made clear that due to a lower TM optical confinement in the wells, a lower number of wells simply causes the level of needed current to increase while not allowing higher levels of nonreciprocity.

The behavior of this slab waveguide was simulated using the first-order perturbation model developed in our previous report. ${ }^{8}$ The details of this model, its derivation, and the validity of the first-order calculations can be found there. Figure 11 in that report shows that a rigorous model and a perturbation formalism achieve the same accuracy for the nonreciprocal Kerr absorption. Besides this numerical proof of the validity of the perturbation formalism, a more physical argument is found when comparing the order of magnitude of the elements of the permittivity tensor of the used MO metals. Using the measured values of Table 1 , it is easily seen that for all three alloy compositions, the magnitude of the off-diagonal element $\left(g=g^{\prime}-j g^{\prime \prime}\right)$ is at least an order of magnitude smaller than the magnitude of the diagonal element, i.e., the dielectric constant $\epsilon_{r}=(n-j \kappa)^{2}$. The last column in Table 1 tabulates $g / \epsilon$. Zaets and Ando prove in Ref. 3 that the transverse MO Kerr terms in the TM dispersion equation are proportional to this ratio [see Eq. (3) in the mentioned reference]. Therefore the values of the last column of Table 1 underline that the introduction of the transverse MO Kerr effect can safely be regarded as a perturbation.

Here we sum up the different perturbation formulas used for the modeling of the SOA-isolator. The details of their derivation can be found in our previous report. ${ }^{8}$

The nonreciprocal absorption shift $\Delta \alpha^{\mathrm{MO}}$ and the specific isolation extinction ratio $I S$ (in decibels per unit length) are given by

$$
\Delta \alpha^{\mathrm{MO}}[\text { in } 1 / \mathrm{cm}]=4 \Im\left[\Delta \beta^{\mathrm{MO}}\right]=-\frac{8 \pi}{Z_{\mathrm{vac}} \lambda} \Re\left[\frac{\int g E_{x}^{(0)} E_{z}^{(0)} \mathrm{d} x}{\int E_{x}^{(0)} H_{y}^{(0)} \mathrm{d} x}\right],
$$


Table 2. Slab Layer Structure to Be Optimized ${ }^{a}$

\begin{tabular}{|c|c|c|c|}
\hline Layer & $\begin{array}{l}\text { Composition } \\
\text { (Dopant, Strain } \varepsilon \text { ) }\end{array}$ & $\begin{array}{l}\text { Material Parameters }(n, g) \\
\left(\text { Bandgap } \lambda_{g}\right)\end{array}$ & Thickness \\
\hline MO layer & $\begin{array}{l}\mathrm{Co}_{90} \mathrm{Fe}_{10} \\
\text { or } \\
\mathrm{Co}_{50} \mathrm{Fe}_{50} \\
\text { or } \\
\mathrm{Fe}\end{array}$ & $\begin{array}{l}n=4.35-i 4.76 \\
g=-1.7+i 1.05 \\
n=3.2-i 4.5 \\
g=-1.7+i 1.7 \\
n=3.82-i 3.5 \\
g=-1.4+i 0.75\end{array}$ & $100 \mathrm{~nm}$ \\
\hline $\begin{array}{l}\text { Absorbing } \\
\text { contact layer }\end{array}$ & $\begin{array}{l}\mathrm{In}_{0.54} \mathrm{Ga}_{0.46} \mathrm{As} \\
\mathrm{Be}: 3 \times 10^{19} \mathrm{~cm}^{-3}\end{array}$ & $\begin{array}{l}n=3.6-i 0.2 \\
1.62 \mu \mathrm{m}\end{array}$ & $15 \mathrm{~nm}$ \\
\hline $\begin{array}{l}\text { Transparent } \\
\text { contact layer }\end{array}$ & $\begin{array}{l}\mathrm{In}_{0.81} \mathrm{Ga}_{0.19} \mathrm{As}_{0.41} \mathrm{P}_{0.59} \\
\mathrm{Be}: 1 \times 10^{19} \mathrm{~cm}^{-3}\end{array}$ & $\begin{array}{l}n=3.37 \\
1.17 \mu \mathrm{m}\end{array}$ & $100 \mathrm{~nm}$ \\
\hline Spacer layer & InP & $\begin{array}{l}n=3.203 \\
0.9 \mu \mathrm{m}\end{array}$ & Optimize \\
\hline Upper SCH & $\mathrm{In}_{0.86} \mathrm{Ga}_{0.14} \mathrm{As}_{0.31} \mathrm{P}_{0.69}$ & $\begin{array}{l}n=3.34 \\
1.05 \mu \mathrm{m}\end{array}$ & Optimize \\
\hline Barrier $(\times 10)$ & $\begin{array}{l}\mathrm{In}_{0.62} \mathrm{Al}_{0.3} \mathrm{Ga}_{0.08} \mathrm{As} \\
\epsilon=+0.6 \%\end{array}$ & $\begin{array}{l}n=3.46 \\
1.05 \mu \mathrm{m}\end{array}$ & $20 \mathrm{~nm}$ \\
\hline $\mathrm{QW}(\times 9)$ & $\mathrm{In}_{0.34} \mathrm{Al}_{0.14} \mathrm{Ga}_{0.52} \mathrm{As}$ & $\begin{array}{l}n=3.57+i k \\
k=0.00483 \ln \left(\frac{6}{N_{w}} \frac{J_{\text {tot }}\left[\text { in } \mathrm{A} / \mathrm{cm}^{2}\right]}{352}\right) \\
1.3 \mu \mathrm{m}\end{array}$ & $10 \mathrm{~nm}$ \\
\hline Lower SCH & $\mathrm{In}_{0.86} \mathrm{Ga}_{0.14} \mathrm{As}_{0.31} \mathrm{P}_{0.69}$ & $\begin{array}{l}n=3.34 \\
1.05 \mu \mathrm{m}\end{array}$ & Optimize \\
\hline Substrate & $\mathrm{InP}$ & $\begin{array}{l}n=3.203 \\
0.9 \mu \mathrm{m}\end{array}$ & \\
\hline
\end{tabular}

${ }^{a}$ Optical constants (at $1300 \mathrm{~nm}$ ) are taken from Ref. 17. The MO constants of the CoFe alloys at this wavelength have been experimentally determined (see Table 1). The expression of the material gain in the QWs is the result of an empirical fit to experimentally measured laser gains (Ref. 16).

$$
\begin{aligned}
I S[\mathrm{in} \mathrm{dB} / \mathrm{cm}] & =-10 \ln \left(P_{\text {out }}^{\mathrm{bw}} / P_{\text {out }}^{\mathrm{fw}}\right) / L \\
& =-10 \ln \left(\exp \left(-\Delta \alpha^{\mathrm{MO}} L\right)\right) / L \\
& =-\frac{80 \pi}{\ln 10 Z_{\mathrm{vac}} \lambda} \Re\left[\frac{\left.\int g E_{x}^{(0)} E_{z}^{(0)} \mathrm{d} x\right]}{\int E_{x}^{(0)} H_{y}^{(0)} \mathrm{d} x}\right] \\
& \approx-\frac{0.3}{\lambda} \Re\left[\frac{\int g E_{x}^{(0)} E_{z}^{(0)} \mathrm{d} x}{\int E_{x}^{(0)} H_{y}^{(0)} \mathrm{d} x}\right],
\end{aligned}
$$

with $E_{x}^{(0)}, E_{z}^{(0)}$, and $H_{y}^{(0)}$ as the field components of the unperturbed TM mode in the metal-clad slab waveguide and $\lambda$ in units of centimeters. Here, $Z_{\mathrm{vac}}\left(=\sqrt{\mu_{0} / \epsilon_{0}}\right)$ is the vacuum impedance, and $I S$ is obtained by calculating the backward-to-forward optical power extinction in decibels when a mode is launched with unit power through a device of length $L$.

The needed transparency current density is found after inverting Eq. (3) once the imaginary part $(\lambda / 4 \pi) G_{\text {transp }}^{\mathrm{QW}}$ the QW index needed to obtain a forward transparent TM mode is known. We have previously shown that the nonreciprocal effect is hardly influenced by the gain present in the active core (see Fig. 7 in our previous report ${ }^{8}$ ). Therefore the needed QW material gain for transparency is found by calculating the TM optical confinement ${ }^{18}$ in the QWs. The TM modal gain is then given by

$$
G_{\mathrm{mod}}^{\mathrm{TM}}(J)=\sum_{i} \Gamma_{i}^{\mathrm{TM}} G_{\mathrm{mat}, i}^{\mathrm{QW}}(J)
$$

with

$$
\Gamma_{i}^{\mathrm{TM}}=\Re\left[\frac{\int_{\mathrm{QW}_{\mathrm{i}}} n_{\mathrm{QW}_{\mathrm{i}}}\left(\left(E_{x}^{(0)}\right)^{2}-\left(E_{z}^{(0)}\right)^{2}\right) \mathrm{d} x}{Z_{\mathrm{vac}} \int E_{x}^{(0)} H_{y}^{(0)} \mathrm{d} x}\right],
$$

with an unambiguous definition for the TM confinement factor and $G_{\mathrm{mat}}^{\mathrm{QW}}(J)$ given by Eq. (3). In most cases, a homogeneous carrier injection is assumed in the QWs, so that a global MQW TM confinement factor can be defined as $\Gamma^{\mathrm{TM}}=\Sigma_{i} \Gamma_{i}^{\mathrm{TM}}$. The forward transparency current density $J_{\mathrm{tr}, \mathrm{fw}}$ for the slab SOA-isolator can now be derived to be

$$
G_{\mathrm{mat}}^{\mathrm{QW}}\left(J_{\mathrm{tr}, \mathrm{fw}}\right)=\frac{\alpha^{(0)}-\Delta \alpha^{\mathrm{MO}} / 2}{\Gamma^{\mathrm{TM}}},
$$

where $\alpha^{(0)}$ is the modal loss of the unperturbed TM mode. This equation will yield $J_{\mathrm{tr}, \mathrm{fw}}$ after inverting Eq. (3).

In other words, the slab SOA-isolator is modeled as a passive metal-clad lossy TM waveguide, and the nonreciprocal absorption effect and the transparency current density are found through perturbation calculations.

\section{B. Optimization Method: a Device Figure of Merit}

As mentioned above, the optimization parameter space is three dimensional and composed of the thicknesses of the InP-spacer layer and those of the quaternary guiding lay- 
ers surrounding the MQW region. To compare the optimality of each point in this parameter space, a realistic and practical device figure of merit (FoM) needs to be defined. This FoM has to be a function of physical external device parameters. For this SOA-isolator, these are the total device length, $L_{A \mathrm{~dB}}$, needed to obtain a certain isolation extinction ratio (say $A \mathrm{~dB}$ ) and the total current needed to achieve forward transparency at this same isolation level, $I_{A \mathrm{~dB}}$. A canonical form for a FoM function to be minimized, is then given by

$$
\mathrm{FoM}=L_{A \mathrm{~dB}}^{m} \times I_{A \mathrm{~dB}}^{n} \quad \text { (with } m, n \text { positive integers). }
$$

The exponents express the relative importance of each device parameter in the optimization function. Relating these device parameters to the modal properties calculated in the perturbation model, i.e., $J_{\mathrm{tr}, \mathrm{fw}}$ and $\Delta \alpha$, the optimization function can be rewritten as a function of the output data of the perturbation calculation,

$$
L_{A \mathrm{~dB}}=\frac{A}{\Delta \alpha}, \quad I_{A \mathrm{~dB}}=\frac{A}{\Delta \alpha} J_{\mathrm{tr}, \mathrm{fw}} \Rightarrow \mathrm{FoM}=A^{m+n} \frac{J_{\mathrm{tr}, \mathrm{fw}}^{n}}{\Delta \alpha^{m+n}} .
$$

We limited our optimization calculations to the cases $m$ $=0, n=1$ and $m=1, n=1$. The former choice minimizes the total absolute current of the device per decibel of isolation, while the latter explicitly seeks a combined optimum of device length and absolute current per decibel of isolation. There is something to be said in favor of both FoM functions. First of all, the I-FoM minimizes the total transparency current needed for a certain isolation level. If the metal is placed sufficiently far from the guiding core, the losses to be compensated for will be low, but also $\Delta \alpha$ will be low. As a result, the device will get longer and because the total current scales with length, it will not be minimized. As a result, the effect has to be increased by placing the metal closer to the guiding core, which will at the same time increase the loss that needs to be compensated for. This trade-off between loss compensation and MO effect is reflected in the $I$-FoM. However, it is not sure whether the MO effect increases in the same manner as the current density, needed to compensate for the losses, when the spacer thickness is decreased. Therefore by also explicitly taking the total length into account in the $L$ $\times I$-FoM (i.e., an extra factor $\Delta \alpha$ in the denominator), another optimal point might be found leading to an even shorter device that needs a transparency current that may not have been proportionally increased. The numerical results presented in Subsection 3.C will prove that this is indeed the case. The actual choice between one or the other design FoM function then depends on what is primordial: an as short as possible device, or a total current that stays below a certain absolute level.

\section{Simulation Results}

Using the above-described waveguide model and optimization algorithm, the device structure of Table 2 has been simulated. In principle, this is a three-dimensional (3D) nonlinear optimization problem that can rather easily be tackled with a conjugated gradient minimization algo- rithm. It has been our experience that the proposed FoM functions exhibit a sufficiently smooth dependence on the three optimization parameters, and that there are no other local extremes. Figure 3 shows a typical example of the scan of the parameter space. Shown here is a contour plot of the $I$-FoM function (expressed in $\mathrm{mA} / \mu \mathrm{m} / \mathrm{dB}$ of isolation) for the layer structure of Table 2 with the equiatomic $\mathrm{Co}_{50} \mathrm{Fe}_{50}$ as the ferromagnetic contact layer and for an InP-spacer thickness of $260 \mathrm{~nm}$. This example serves to illustrate the smoothness of the FoM function and the existence of a single optimum for each InP-spacer thickness. These simulations have been performed using a freely available $\mathrm{C}++$-based photonic component modeling framework developed by our coauthors for the unperturbed waveguide modal calculations. ${ }^{19}$ The first-order perturbation integrals have been numerically integrated with an extended Simpson formula.

Both FoM functions have been minimized in equally spaced $\left(t_{\mathrm{SCH}, u}, t_{\mathrm{SCH}, l}\right)$ planes (i.e., $t_{\mathrm{InP}}=$ constant) in the $3 \mathrm{D}$ parameter space, and this has been done for each of the three CoFe-alloy compositions. The results of this minimization are plotted in Fig. 4(a) $I$ and Fig. 4(b) $L \times I$. The minimum in each of these plots thus corresponds to the absolute minimum of the FoMs throughout the entire 3Dparameter space. These are indicated by arrows.

Figure 5 plots the values for the isolator length and the needed current (both per decibel of isolation extinction) corresponding to the points in Fig. 4. The arrows in these figures correspond the absolute minima of the two FoM functions. Table 3 summarizes the results of the optimization calculations. It enumerates the optimum thicknesses within the $3 \mathrm{D}$ parameters space at the absolute minima of both FoM functions (and for the three alloy compositions) and the corresponding SOA-isolator performance at these design points, namely, the absolute current (in $\mathrm{mA} / \mu \mathrm{m}$ width of the active region and per decibel of isolation) and the total length (in $\mathrm{mm} / \mathrm{dB}$ of isolation).

\section{DISCUSSION}

The results of these optimization calculations reveal several important characteristics of the novel isolator con-

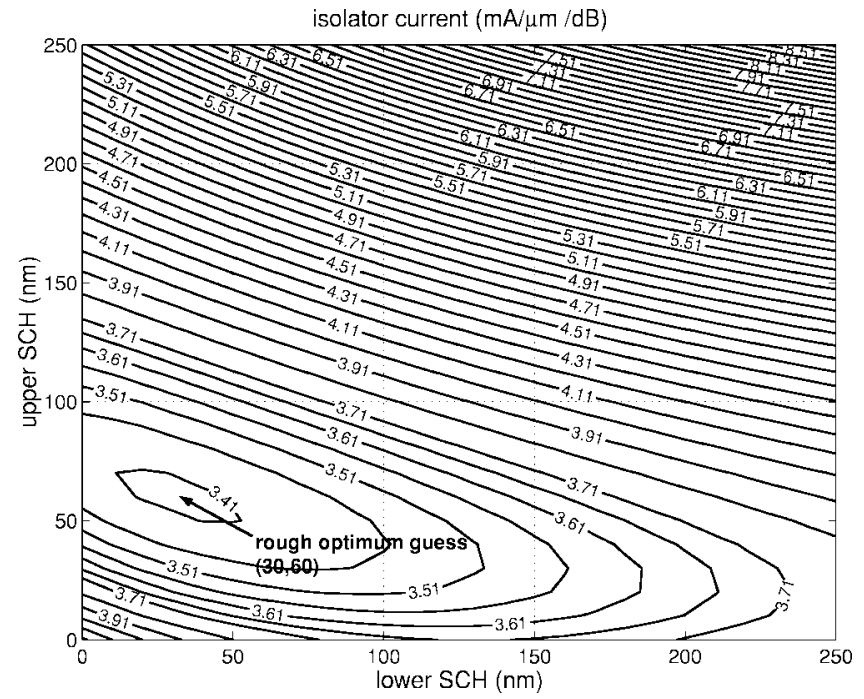

Fig. 3. I-FoM scan of the SCH parameter space (at $t_{\mathrm{InP}}$ $=260 \mathrm{~nm})$ for $\mathrm{a} \mathrm{Co}_{50} \mathrm{Fe}_{50}$ SOA-isolator. 

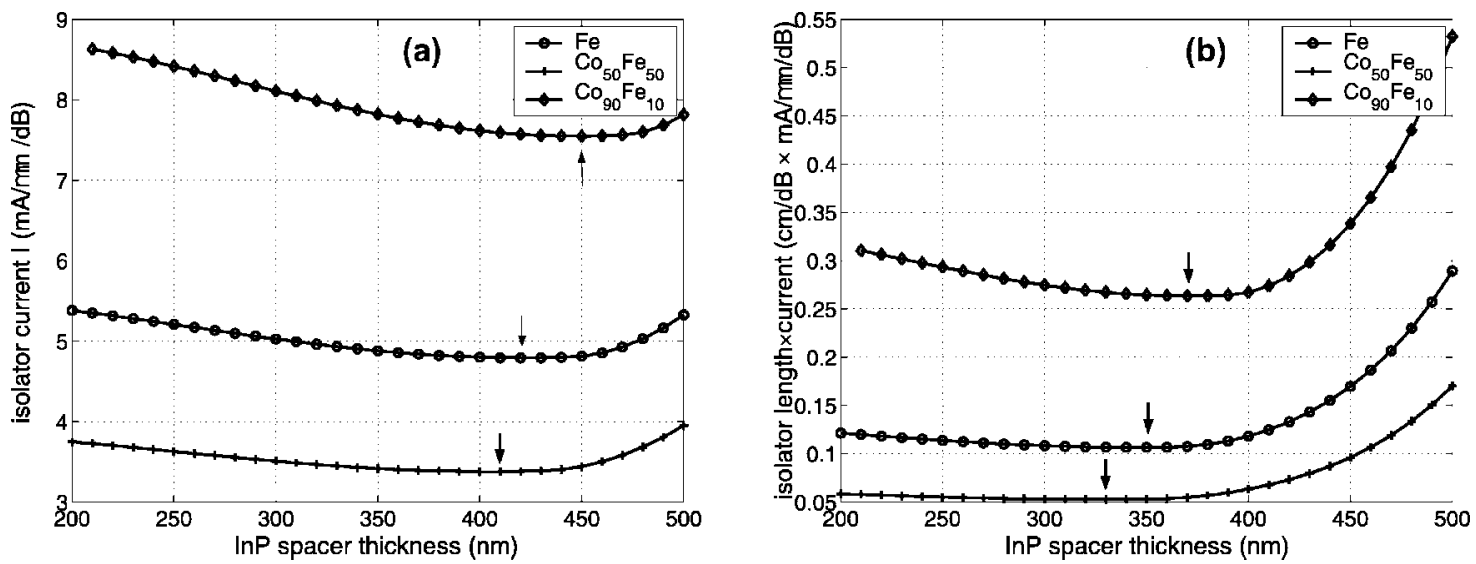

Fig. 4. Optimum values of the two considered FoMs (a) $I$, (b) $L \times I$ within each considered $\left(t_{\mathrm{SCH}, l}, t_{\mathrm{SCH}, u}\right)$ plane (i.e., $t_{\mathrm{InP}}=C$ with $C$ $=500, \ldots, 200 \mathrm{~nm})$. The arrows indicate the absolute minima.
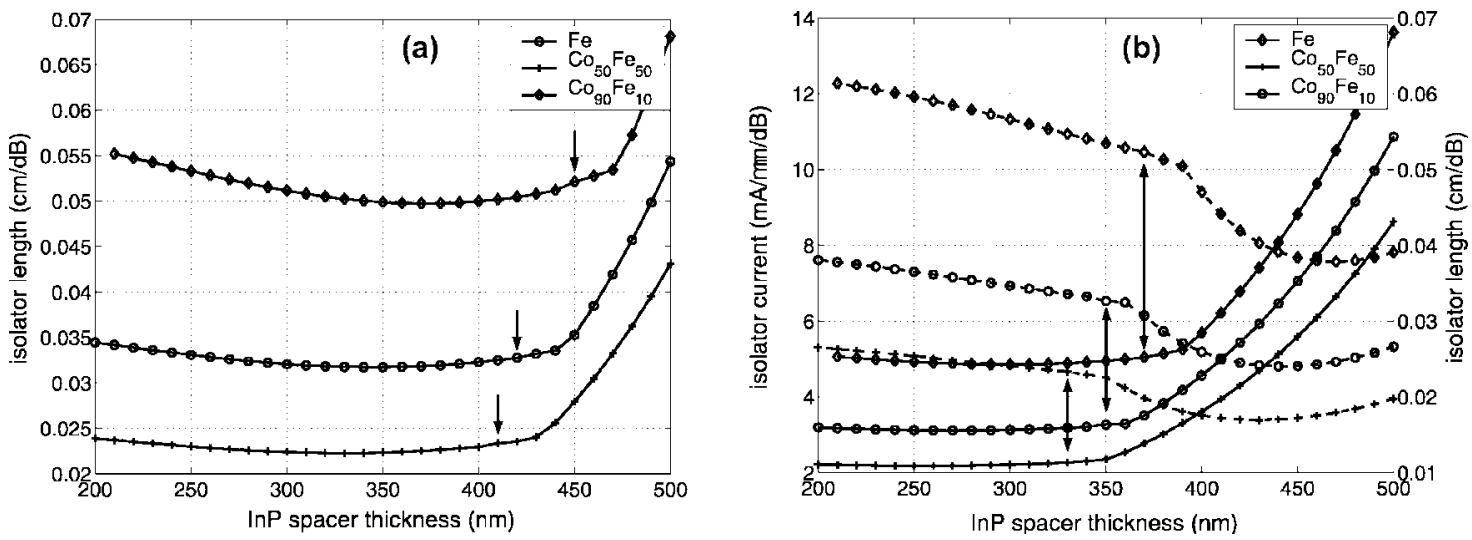

Fig. 5. Isolator length $(\mathrm{cm} / \mathrm{dB})$ (solid curve) and total isolator current $(\mathrm{mA} / \mu \mathrm{m} / \mathrm{dB}$ ) (dashed curve) corresponding to the design points of Figs. 4(a) $I$, 4(b) $L \times I$.

Table 3. Results of the Optimization Calculations of the SOA-Isolator Layer Structure

\begin{tabular}{cccccc}
\hline & \multicolumn{3}{c}{ FoM $I$} \\
\cline { 2 - 6 } & $\begin{array}{c}t_{\mathrm{InP}} \\
(\mathrm{nm})\end{array}$ & $\begin{array}{c}t_{\mathrm{SCH}, 1} \\
(\mathrm{~nm})\end{array}$ & $\begin{array}{c}t_{\mathrm{SCH}, \mathrm{u}} \\
(\mathrm{nm})\end{array}$ & $\begin{array}{c}I \\
(\mathrm{~mA} /(\mu \mathrm{m} \times \mathrm{dB}))\end{array}$ & $\begin{array}{c}L \\
(\mathrm{~mm} / \mathrm{dB})\end{array}$ \\
\hline $\mathrm{Fe}$ & 420 & 14.8 & 14.8 & 4.793 & 0.326 \\
$\mathrm{Co}_{50} \mathrm{Fe}_{50}$ & 410 & 13.5 & 14.5 & 7.378 & 0.233 \\
$\mathrm{Co}_{90} \mathrm{Fe}_{10}$ & 450 & 12.5 & 13.5 & & 0.521 \\
& & & FoM $L \times I$ & 6.526 & \\
$\mathrm{Fe}$ & 350 & 16 & 16 & 4.658 & 0.163 \\
$\mathrm{Co}_{50} \mathrm{Fe}_{50}$ & 330 & 12 & 15 & 10.458 & 0.113 \\
$\mathrm{Co}_{90} \mathrm{Fe}_{10}$ & 370 & 10.7 & 13.2 & 0.252 \\
\hline
\end{tabular}

cept. In this section, we discuss the attainable performances of the device and its design tolerances. The section is concluded by a deeper look at what the physical limitations of the device concept are, and presents some suggestions for possible improvements.

\section{A. Performance}

Looking at the FoM plots of Fig. 4, it can be seen that starting from a rather thick InP-spacer thickness of $500 \mathrm{~nm}$, the device performance can be considerably improved by placing the metal closer to the guiding core (and simultaneously readjusting the thicknesses of the
SCH layers). As explained above, for the I-FoM [Fig. 4(a)], this is the result of an increasing $\Delta \alpha$ leading to a reduction of the device length and thus of the total current, while at the same time the loss to be compensated for does not increase as fast as $\Delta \alpha$. Continuing to decrease $t_{\text {InP }}$ below a certain optimal thickness, this trend is inverted, and the loss to be compensated for increases faster than $\Delta \alpha$.

For the $L \times I$-FoM [Fig. 4(b)], the explicit inclusion of the device length in the merit function allows for pushing somewhat further: $t_{\mathrm{InP}}$ can be further decreased beyond the point of optimal total current because the increase in 
$I$ beyond this point can still be compensated for by a further decrease in the total device length $L$ (due to a higher $\Delta \alpha$ ). But also here, below some optimal InP thickness, the total current increases faster than the device length decreases.

Table 3 illustrates that the use of the $L \times I$-FoM leads to an optimal design point with a shorter device length than when using the $I$-FoM. This obviously comes at the price of a higher total current. However, the values in this table show that the relative increase in total current is lower than the relative decrease in device length. For $\mathrm{Co}_{50} \mathrm{Fe}_{50}$, the current increases by $\approx 30 \%$, while the length decreases by $\approx 50 \%$. Whether therefore the minimal $L \times I$ design is to be preferred above the minimal $I$ design, depends on the consideration if the higher total absolute current can be sustained by the SOA-isolator, and if power consumption is a less important issue than device dimensions. In any case, with the tabulated optimization values, one finds that the best obtainable performance for, say a $25 \mathrm{~dB}$ SOA-isolator using this MO metal alloy system, is either a device of $5.825 \mathrm{~mm}$ long and consuming $84.5 \mathrm{~mA}$ (per micrometer ridge width) or a device of $2.825 \mathrm{~mm}$ long and consuming $116.5 \mathrm{~mA}$ (per micrometer ridge width).

Another important conclusion of these calculations is that comparing the merit functions between the different alloy compositions (Fig. 4) proves that the equiatomic alloy composition $\mathrm{Co}_{50} \mathrm{Fe}_{50}$ easily outperforms pure $\mathrm{Fe}$ and Co-rich alloy compositions. This is contrary to recent claims by Shimizu and Nakano. ${ }^{20,21}$ that a MO Fe contact layer is best suited for this type of nonreciprocal device.

What is also striking is that pure Fe outperforms $\mathrm{Co}_{90} \mathrm{Fe}_{10}$, even though the ellipsometric results summarized in Table 1 clearly indicate a much lower gyrotropic dichroism [i.e., $\Im(g)$ ] for $\mathrm{Fe}$ than for $\mathrm{Co}_{90} \mathrm{Fe}_{10}$. One might argue that the FoMs plotted in Fig. 4 include the transparency current density [see Eq. (8) with $n=1$ ], and that as a result, the stronger absorbing $\mathrm{Co}_{90} \mathrm{Fe}_{10}\left(\kappa_{\mathrm{Co}_{90} \mathrm{Fe}_{10}}\right.$ $=1.38 \kappa_{\mathrm{Fe}}$ ) explains the worse merit functions. However, when calculating only the nonreciprocal absorption $\Delta \alpha$, we have observed that Fe generally does better than the Co-rich alloy, despite its lower MO dichroism. This indicates that the value of $\Delta \alpha$ is the result of a more complicated interplay between the gyrotropy parameter and the properties of the unperturbed field profile and is not so simply directly related to the dichroic properties of the used ferromagnetic metal. This has already been remarked on by Zayets and Ando in Ref. 22, where they correctly indicated that the properties of the layer below the MO metal influence the magnitude of the magnetoreflectivity at the MO interface. However, they did not indicate the reason for this influence. We have found that this is due to the obtainment of a better (or worse) phase accordance between the phase of the gyrotropy parameter $g$ and that of the electric-field component $E_{x}$ at the interface. In Subsection 4.C, a closed analytical expression is derived that clearly illustrates this. In any case, our calculations combined with our ellipsometric experiments show that within the CoFe-alloy system, there exists a clear optimum composition for this MO waveguide application, and that at $1300 \mathrm{~nm}$, this optimum is at or close to the equiatomic composition.

\section{B. Tolerances}

\section{Layer Thicknesses}

An important aspect of any design calculation is the tolerance of the predicted performance with respect to the design parameters. To get a clear idea of the different tolerances, the two merit functions are evaluated in the neighborhood of the optimum design points for the equiatomic alloy. This is illustrated in Fig. 6. Here, the merit function is contour plotted as a function of two of the three design parameters with the third one fixed to its optimum value (along Table 3). Each subsequent contour corresponds to an increase of $1 \%$ of the minimum of the considered FoM function.

These figures teach us that the optimally designed device is robust with respect to layer thickness variations. First of all, it is clear that at the optimum value for the InP-spacer thickness [see the contours in Figs. 6(a) and $6(d)]$ the design performance is very tolerant toward the thicknesses of the guiding layers, and that at this $t_{\mathrm{InP}, \mathrm{opt}}$, these layers show very low correlation. The SCH thicknesses may vary anywhere between 0 and $50 \mathrm{~nm}$ without causing an increase of more than $10 \%$ of the FoM optimum. However, this paints a too optimistic picture. Indeed, when looking at the FoM contours, also taking $t_{\text {InP }}$ into account, it becomes clear that the device actually has a very small dependence on the thickness of the lower $\mathrm{SCH}$ layer, as can be seen from the horizontally oriented contours in Figs. 6(b) and 6(e). This could already have been remarked on when looking at Fig. 3 where it is seen that the gradient of the FoM is much more pronounced in the direction of the upper SCH layer. This is a direct consequence of the asymmetry of the slab waveguide problem and the well-known plasmonic amplitude enhancement of a TM mode at the interface between a strong absorbing layer and a dielectric. ${ }^{23}$ As a result, the lower SCH has much less effect in controlling the balance between confinement in the active region and confinement in the MO metal. Finally, the fact that the performance of this device is almost entirely controlled by just the two thicknesses $t_{\mathrm{InP}}$ and $t_{\mathrm{SCH}}$ is even more underlined by the contour plots (c) and (f) in Fig. 6. Here, it can be clearly seen that $t_{\mathrm{InP}}$ and $t_{\mathrm{SCH}}$ are strongly correlated, and that their optimum values (within $10 \%$ of the minimum) lay on a straight line in this parameter plane.

In a rough approximation, one can conclude that the design of the SOA-isolator using the proposed merit functions is very robust with respect to layer thicknesses. The optimum design points (within $10 \%$ of the minimum) are independent of $t_{\mathrm{SCH}, 1}$ and lay on a quasi-straight line in the $\left(t_{\mathrm{InP}}, t_{\mathrm{SCH}, \mathrm{u}}\right)$ plane described by $t_{\mathrm{InP}}+t_{\mathrm{SCH}, \mathrm{u}}=C$, with $C \approx 425$ or $350 \mathrm{~nm}$ for, respectively, the $I$ and the $L \times I$-FoM. This last expression could be used as a design rule for a first quasi-optimized device.

\section{Operation Wavelength}

A second tolerance aspect to be studied for this device is its wavelength sensitivity. Here, it has to be remarked on that for standard MQW SOAs, the gain spectrum is traditionally not much wider than $20-30 \mathrm{~nm}$. This is a variation of less than $20 \mathrm{meV}$. It is safe to assume that neither the optical nor the MO indices of the CoFe alloys 

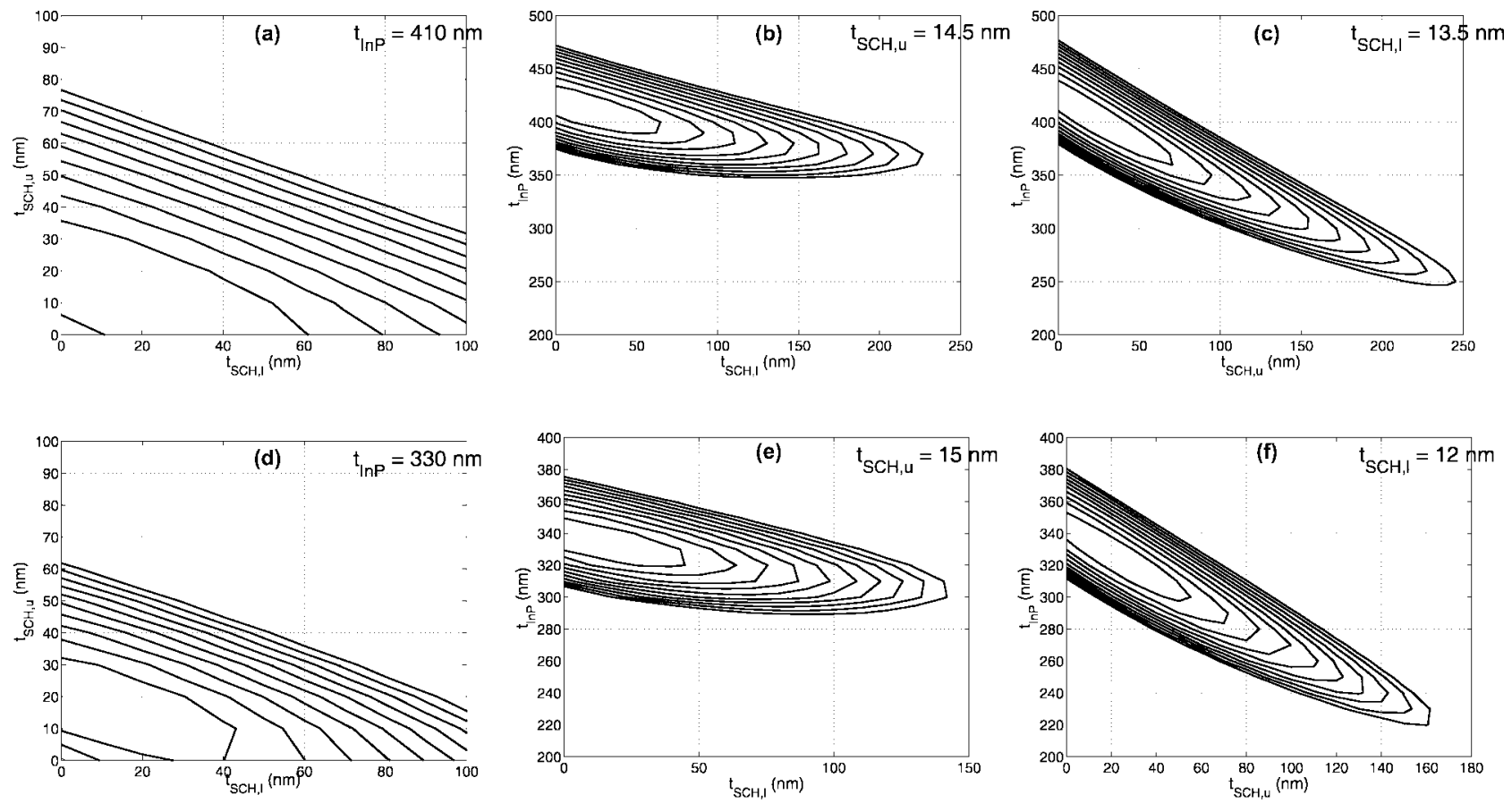

Fig. 6. Equi-FoM contours for the $\mathrm{Co}_{50} \mathrm{Fe}_{50}$-clad SOA isolator in the neighborhood of the optimum design point. The outer contour represents an increase of $10 \%$ of the absolute minimum value; the spacing of the contours is $1 \%$. Subplots (a) (b), and (c) are for the $I$-FoM function and for the optimum thickness of, respectively, (a) InP layer, (b) upper SCH layer, and (c) lower guiding layer. The same applies for the subplots (d)-(f) but for the $L \times I$ FoM.

will vary importantly over such a small energy interval. If we hypothetically assume a homogeneous gain [given by the empirical relationship of Eq. (3)] over a $25 \mathrm{~nm}$ wide wavelength window at around $1300 \mathrm{~nm}$ and recalculate, for these wavelengths, the FoMs of Eq. (9) with the optimized layer structure at $1300 \mathrm{~nm}$, an increase of less than $5 \%$ of the FoM is found. This indicates that the optimization calculation is also very robust with respect to wavelength.

\section{Metal Indices}

A final tolerance aspect to be studied is the influence of the uncertainties on the experimentally determined MO parameters. While standard ellipsometry easily achieves accuracies of just a few percent on the optical indices, an accuracy of $\sim 10 \%$ is more common for the MO constants. If we can safely assume that the optical constants are correct, then the influence of variations in the value of the MO index can be very easily studied in our perturbation model since the perturbation integral of Eq. (4) does not have to be re-evaluated. This is because it is assumed that the MO perturbation is constant throughout the metal. The nonreciprocal absorption shift can be written as

$$
\Delta \alpha=\Re(g A),
$$

with

$$
A=-\frac{4 k_{0}}{Z_{\mathrm{vac}}} \frac{\int_{\text {metal }} E_{x}^{(0)} E_{z}^{(0)} \mathrm{d} x}{\int E_{x}^{(0)} H_{y}^{(0)} \mathrm{d} x},
$$

with $k_{0}=2 \pi / \lambda$. In this equation, the variable $A$ only depends on unperturbed waveguide modal properties. Assuming that the modal profiles have been properly normalized, the denominator of $A$ will equal 2 , and the complex behavior of $A$ is entirely determined by the field integral $\int_{\text {metal }} E_{x}^{(0)} E_{z}^{(0)} \mathrm{d} x$. In a lossy metal-clad waveguide, the modal field profiles will exhibit a nonnegligible phase curvature in the neighborhood of and within the absorbing layer. In a transparent or a "limited-loss" slab waveguide, the phase front curvature of a TM mode is absent or very small, and the phase difference between $E_{x}$ and $E_{z}$ is always equal or close to $\pi / 2$. The variable $A$ would then be predominantly imaginary, and Eq. (10) teaches us that $\Delta \alpha$ is dominated by the imaginary (dichroic) part of the gyrotropy constant, $\Im(g)$. In the present case however, the phase front curvature will be large, and $\angle\left(E_{x} E_{z}\right) \neq \pi / 2$. This indicates that $\Delta \alpha$ will be influenced by both the real and imaginary parts of $g$. Of course, this is a direct consequence of the fact that the MO layer has a very high optical loss, causing the important phase front curvature. All of the above is illustrated in Fig. 7 where the variations of $\Delta \alpha$ of the $L \times I$ design point are plotted when the gyrotropy parameter is varied between the accuracy limits of Table 1 . It is clear that both $\Re(g)$ and $\Im(g)$ contribute to $\Delta \alpha$ with almost the same strength (which means that $\angle A \approx \pi / 4)$. It can thus be concluded that the tolerance of the device with respect to inaccuracies in the measured MO parameter entirely depends on the phase front curvature of the unperturbed TM mode, which in turn depends on the complex refractive index of the metal and the ensuing modal effective index. As this curvature is, in general, not negligible in a metal-clad waveguide, it can be said that the relative uncertainty on the device perfor- 


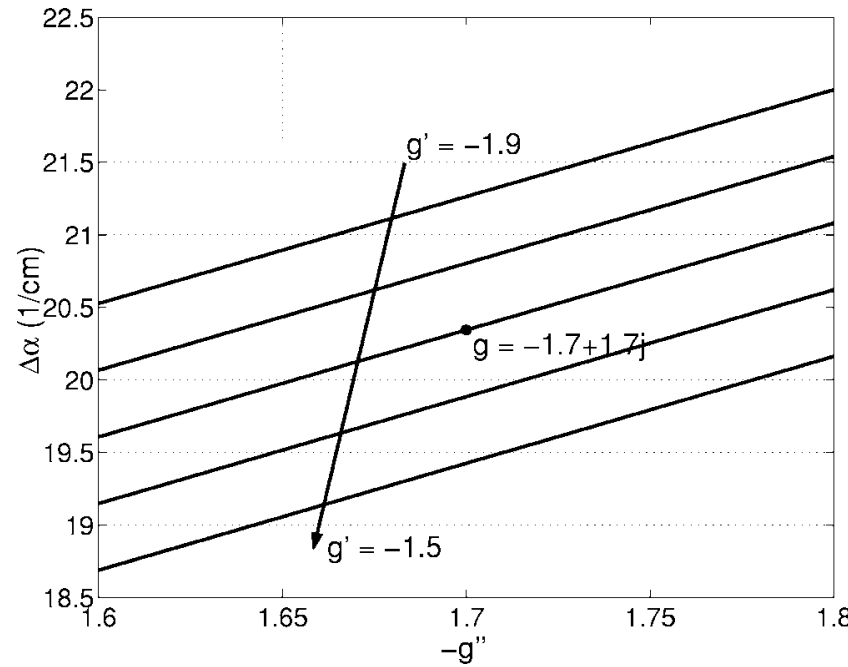

Fig. 7. Variations of the nonreciprocal absorption shift (in $1 / \mathrm{cm}$ ) in terms of the measurement accuracy of the dichroic part of the gyrotropy parameter of $\mathrm{Co}_{50} \mathrm{Fe}_{50}$ and for different values of $\Re(g)$ (see Table 1). The black dot indicates the optimum $L \times I$ design point obtained with the values of Table 1 .

mance will never be higher than the highest relative uncertainty on either $\mathfrak{R}(g)$ or $\mathfrak{I}(g)$.

If we take this analysis one step further, an analytical expression can be derived that indicates what fundamentally limits the nonreciprocal absorption effect in this $\mathrm{MO}$ metal-clad slab waveguide. Equation (10) makes clear that $\Delta \alpha$ is maximized when

$$
\angle(A)=\left\{\begin{array}{l}
-\angle(g) \\
\pi-\angle(g)
\end{array} .\right.
$$

If it is assumed that the unperturbed TM modal profiles are adequately normalized $\left(\int E_{x}^{(0)} H_{y}^{(0)} \mathrm{d} x=2\right)$, then the complex behavior of $A$ is determined by the field integral $\int_{\text {metal }} E_{x}^{(0)} E_{z}^{(0)} \mathrm{d} x$. For a slab waveguide, this integral can be very easily evaluated. Indeed, within a homogeneous region, the Maxwell divergence equation $\nabla \cdot\left(\epsilon \mathbf{E}^{(0)}\right)=0$ allows us to write for a TM mode,

$$
E_{z}^{(0)}=\frac{-j}{k_{0} n_{\mathrm{eff}}} \frac{\mathrm{d} E_{x}^{(0)}}{\mathrm{d} x}
$$

Using Eq. (12) and assuming that the metal is sufficiently thick to be optically opaque, the field integral evaluates to

$$
\begin{aligned}
\int_{\text {metal }} E_{x}^{(0)} E_{z}^{(0)} \mathrm{d} x & =\frac{-j}{2 k_{0} n_{\text {eff }}} \int_{\text {metal }} \frac{\mathrm{d}\left(E_{x}^{(0)}\right)^{2}}{\mathrm{~d} x} \mathrm{~d} x \\
& =\frac{j}{2 k_{0} n_{\text {eff }}}\left(E_{x}^{(0)}\right)^{2}\left(x_{m+}\right),
\end{aligned}
$$

with $x_{m+}$ as the $x$ coordinate infinitesimally above the metal interface. So that finally [using Eq. (10)], the phase angle of $A$ is given by

$$
\angle A=\angle\left(\frac{-j}{Z_{\mathrm{vac}} n_{\mathrm{eff}}}\left(E_{x}^{(0)}\right)^{2}\left(x_{m+}\right)\right) \approx-\frac{\pi}{2}+2 \angle E_{x}^{(0)}\left(x_{m+}\right),
$$

where the last approximation refers to the fact that the imaginary part of the effective index of the guided mode is much smaller than its real part so that its phase angle is quasi-zero. Using this result, Eq. (10) then reduces to

$$
\Delta \alpha=\frac{|g|\left|E_{x}^{(0)}\left(x_{m+}\right)\right|^{2}}{Z_{\mathrm{vac}}\left|n_{\mathrm{eff}}\right|} \sin \left(\angle g+2 \angle E_{x}^{(0)}\left(x_{m+}\right)\right),
$$

which maximizes when $\angle E_{x}^{(0)}\left(x_{m+}\right)=\pi / 4-\frac{1}{2} \angle g+m \pi / 2$ and annulates when $\angle E_{x}^{(0)}\left(x_{m+}\right)=m \pi / 2-\frac{1}{2} \angle g$. This last form for the nonreciprocal absorption shift indicates very clearly that in order to get an optimal isolation effect in this type of device, there are three factors that have to work in unison: (1) the amplitude of the gyrotropy $|g|$ has to be as high as possible, (2) the amplitude of the $x$ component of the electric field of the unperturbed mode at the metal interface has to be as high as possible, and (3) the phase angle of this component (or thus the phase front curvature) at this interface has to show a good specific relationship with the gyrotropy phase angle, $\angle g$.

In particular, this last condition limits the possible performance of the device. Contrary to physical intuition, it is not sufficient to have a MO metal available with very strong MO effects. The impact of the presence of the metal on the guided modal properties through its isotropic parameters is almost as influential on the device performance. And not primordially because it will cause modal absorption that has to be compensated for, but in the first place, through its influence on the amplitude of the electric field at the metal interface and its phase curvature. It is because of the above-described effects that, for example, the Co-rich alloy $\mathrm{Co}_{90} \mathrm{Fe}_{10}$ causes a smaller nonreciprocal absorption effect than pure Fe, even though $\left|g_{\mathrm{Co}_{90} \mathrm{Fe}_{10}}\right|=1.25\left|g_{\mathrm{Fe}}\right|$.

The influence of the real and imaginary parts of the metal refractive index on the phase front curvature and the amplitude of the electric field at the metal interface is not so straightforward to classify. It is clear that these influences will, among others, be heavily dominated by the surface-plasmon-polariton effects taking place at the semiconductor-MO metal interface, and these are influenced both by the ratio of the real and imaginary parts of the metal refractive index and by their magnitude. Work is underway to study these effects in more detail. In any case, this section has made it abundantly clear that the nonreciprocity effects in ferromagnetic metal-clad waveguides is influenced by much more than just the gyrotropy constant. There are extra subtleties involved also caused by the isotropic constants of the metal. This was previously qualitatively noted by Zayets and Ando, ${ }^{22}$ and with our derived Eq. (15), this can now also be quantified.

\section{Possible Improvements}

In this final subsection, we would like to point out some possible directions for improvement of the device behavior. Still it needs to be underlined that the performance of the device presented here is well within the limits of what 
is practically acceptable and, moreover, has also been confirmed experimentally by our coauthors. ${ }^{7}$

First of all, it is not entirely clear whether the concept can profit from using a higher number of QWs. Up till now, this number has always been limited to nine. It has been assessed that a lower number of wells decreases the optical confinement too much to achieve reasonable operation current levels. A higher number of wells might allow for smaller thicknesses for the InP spacer and hence higher $\Delta \alpha$. However, as the number of wells increases, the MQW transparency current density also scales along while the increase in optical TM MQW confinement will show a sublinear increase. At the same time, there are experimental indications, as mentioned in Subsection 2.B, that an increasing number of wells might suffer from a reduction in internal quantum efficiency.

Second, up till now the optimization parameters have been limited to the thicknesses of the three most important layers in the structure, the spacer layer, and the two guiding layers. It has not been investigated whether a composition variation (and thus a refractive index variation) of these layers might advantageously influence the performance of the device. Again, one must remark here that the composition of these layers is not entirely a free parameter. The bandgap of the guiding layers plays a role in the quantum-mechanical problem of the carrier recombination in the QWs and thus in the gain behavior of the device. Only the composition of the spacer layer might be varied. Replacing it by a lower bandgap InP-latticematched composition will increase its refractive index and might have a profound impact on the guiding problem near the metal, possibly causing an increase of the ratio $\Delta \alpha / J_{\text {transp. }}$ This needs to be studied.

Finally, it should be remarked that varying the thickness of the MO metal will not bring much improvement to the device performance. It has been remarked in our previous paper ${ }^{8}$ that due to an interference effect there is a maximum in $\Delta \alpha$ when the metal thickness is varied. However, this increase is not spectacular and is also accompanied by a similar increase in needed isolator current.

\section{CONCLUSION}

In this paper, we have given a detailed overview of our design work on a novel concept for a monolithic integrated optical waveguide isolator operating at $1.3 \mu \mathrm{m}$. The concept is based on a nonreciprocal MO Kerr effect-based absorption shift of the guided TM modes when a standard InP-based waveguide is clad by a transversely magnetized ferromagnetic metal layer. By using an active SOA layout for the waveguide and using the ferromagnetic metal also as an ohmic $p$-type contact for the underlying SOA structure, the optical losses of the guided TM mode caused by the metal layer can be compensated for in one propagation direction, while net loss remains for the opposite propagation direction. We have presented an optimization method of the transversal layer structure that is based on the introduction of two merit functions, minimal current consumption or minimal product of device length and current consumption. This optimization strategy has been used to study a CoFe-clad version of the proposed SOA-isolator. The conclusions of these optimization de- signs are threefold. (1) Within the CoFe alloy system, the equiatomic $\mathrm{Co}_{50} \mathrm{Fe}_{50}$ composition strongly outperforms the other compositions. (2) The optimal design is very robust with respect to variations of layer thickness and wavelength. And the accuracy of the predicted performance (in terms of uncertainties in the MO parameters) is at least better than the lowest accuracy of the complex parts of $g$. (3) This isolator concept with the layer structure of Table 2 can, when optimized along this procedure, provide practical isolation performance for $1300 \mathrm{~nm}$ applications with acceptable device parameters $(25 \mathrm{~dB}$ isolation, $2.825 \mathrm{~mm}$ long, and consuming $116.5 \mathrm{~mA} / \mu \mathrm{m}$ ridge width).

Finally, a new analytical insight has been gained in how the MO nonreciprocal absorption shift in this device is governed by an interplay between both the complex gyrotropy constant and the complex refractive index of the used ferromagnetic metal. This is an important theoretical assessment as it was previously believed that the device performance only depends on the dichroic part of the gyrotropy constant and the optical absorption of the MO metal. This improved insight will be useful in identifying other even more suitable MO materials for this application.

\section{ACKNOWLEDGMENTS}

This work has been performed within the framework of an European IST research project, IST-ISOLASER. The authors thank B. Dagens and J. Decobert of Alcatel Thales III-V Laboratory, France for their work on the development of the tensile-strained InAlGaAs MQW material. P. Bienstman of the Department of Information Technology (INTEC) at Ghent University, Belgium is acknowledged for his continuous effort in developing the photonic modeling framework CAMFR and making it freely available.

M. Vanwolleghem's e-mail address is mathias.vanwolleghem@ief.u-psud.fr.

\section{REFERENCES}

1. H. Dötsch, N. Bahlmann, O. Zhuromskyy, M. Hammer, L. Wilkens, R. Gerhardt, P. Hertel, and A. F. Popkov, "Applications of magneto-optical waveguides in integrated optics: review,” J. Opt. Soc. Am. B 22, 240-253 (2005).

2. H. Yokoi, T. Mizumoto, N. Shinjo, N. Futakuchi, and Y. Nakano, "Demonstration of an optical isolator with a semiconductor guiding layer that was obtained by use of a nonreciprocal phase shift," Appl. Opt. 39, 6158-6164 (2000).

3. W. Zaets and K. Ando, "Optical waveguide isolator based on nonreciprocal loss/gain of amplifier covered by ferromagnetic layer," IEEE Photon. Technol. Lett. 11, 1012-1014 (1999).

4. M. Takenaka and Y. Nakano, "Proposal of a novel semiconductor optical waveguide isolator," in Proceedings of the 11th International Conference on Indium Phosphide and Related Materials (IEEE, 1999), pp. 298-292.

5. J. M. Hammer, J. H. Abeles, and D. J. Channin, "Polycrystalline-metal-ferromagnetic optical waveguide isolator (POWI) for monolithic-integration with diode-laser devices,” IEEE Photon. Technol. Lett. 9, 631-633 (1997).

6. M. Vanwolleghem, W. Van Parys, D. Van Thourhout, R. Baets, F. Lelarge, O. Gauthier-Lafaye, B. Thedrez, R. 
Wirix-Speetjens, and L. Lagae, "Experimental demonstration of nonreciprocal amplified spontaneous emission in a CoFe clad semiconductor optical amplifier for use as an integrated optical isolator," Appl. Phys. Lett. 85, 3980-3982 (2004).

7. W. Van Parys, B. Moeyersoon, D. Van Thourhout, R. Baets, M. Vanwolleghem, B. Dagens, J. Decobert, O. Le Gouezigou, D. Make, R. Vanheertum, and L. Lagae, "Transverse magnetic mode nonreciprocal propagation in an amplifying AlGaInAs/InP optical waveguide isolator," Appl. Phys. Lett. 88, 071115 (2006).

8. K. Postava, M. Vanwolleghem, D. Van Thourhout, R. Baets, Š. Višňovský, P. Beauvillain, and J. Pištora, "Modeling of a novel InP-based monolithically integrated magneto-optical waveguide isolator," J. Opt. Soc. Am. B 22, 261-273 (2005).

9. A. K. Zvezdin and V. A. Kotov, "Polarized light and gyrotropic media," in Modern Magnetooptics and Magnetooptical Materials (Institute of Physics, 1997), pp. 19-32.

10. C. Vassallo, "1993-1995 Optical mode solvers," Opt. Quantum Electron. 29, 95-114 (1997).

11. J. Fujita, M. Levy, R. M. Osgood, Jr., L. Wilkens, and H. Dötsch, "Waveguide optical isolator based on Mach-Zehnder interferometer," Appl. Phys. Lett. 76, 2158-2160 (2000).

12. H. Yokoi, T. Mizumoto, and Y. Shoji, "Optical nonreciprocal devices with a silicon guiding layer fabricated by wafer bonding," Appl. Opt. 42, 6605-6612 (2003).

13. W. Van Parys and M. Vanwolleghem, "Report on I-V characteristics of $\mathrm{CoFe}$ contacted device structure ressembling closely the final structure," IST-ISOLASER project Deliverable D11, available at http:// pcphotonics2.intec.ugent.be/isolaser/.

14. A. Lesuffleur, M. Vanwolleghem, P. Gogol, B. Bartenlian, P. Beauvillain, J. Harmle, L. Lagae, J. Pištora, K. Postava, Š. Višňovský, and R. Wirix-Speetjens, "Magneto-optical parameters of $\mathrm{Co}_{90} \mathrm{Fe}_{10}$ and $\mathrm{Co}_{50} \mathrm{Fe}_{50}$ ferromagnetic thin films for $1.3 \mu \mathrm{m}$ integrated isolator," J. Magn. Magn. Mater. 305, 284-290 (2006).

15. R. Atkinson and P. H. Lissberger, "Sign conventions in magneto-optical calculations and measurements," Appl. Opt. 31, 6076-6081 (1992).

16. J. Decobert, N. Lagay, C. Cuisin, B. Dagens, B. Thedrez, and F. Laruelle, "MOVPE growth of AlGaInAs-InP highly tensile-strained MQWs for $1.3 \mu \mathrm{m}$ low-threshold lasers," J. Cryst. Growth 272, 543-548 (2004).

17. B. Jensen, "Calculation of the refractive index of compound semiconductors below the band gap," in Handbook of Optical Constants of Solids II, E. D. Palik, ed. (Academic, 1991), pp. 125-149.

18. T. D. Visser, B. Demeulenaere, J. Haes, D. Lenstra, R. Baets, and H. Blok, "Confinement and modal gain in dielectric waveguides,” J. Lightwave Technol. 14, 885-887 (1996).

19. P. Bienstman and R. Baets, "Optical modelling of photonic crystals and VCSELs using eigenmode expansion and perfectly matched layers," Opt. Quantum Electron. 33, 327-341 (2001).

20. H. Shimizu and Y. Nakano, "Fabrication a TE mode InGaAsP active waveguide optical isolator based on the nonreciprocal loss shift," in Magneto-Optical Materials for Photonics and Recording, K. Ando, W. Challener, R. Gambino, and M. Levy, eds., Proc. SPIE 834, 834-839 (2005).

21. H. Shimizu and Y. Nakano, "First demonstration of TE mode nonreciprocal propagation in an InGaAsP/InP active waveguide for an integratable optical isolator," Jpn. J. Appl. Phys. Part 2 43, L1561-L1563 (2004).

22. V. Zayets and K. Ando, "Isolation effect in ferromagneticmetal/semiconductor hybrid optical waveguide," Appl. Phys. Lett. 86, 261105 (2005).

23. H. Raether, Surface Plasmons-on Smooth and Rough Surfaces and on Gratings, Vol. III of Springer Tracts in Modern Physics (Springer-Verlag, 1988). 